from 0.28 per cent. to 0.10 per cent. and the total acidity from 80 to 40 ; but, nevertheless, jejunal ulceration ensued, even after partial gastrectomy, and in spite of symptomatic relief from medical treatment ran a progressive course to final perforation. In Case II, despite a partial gastrectomy, the final level of acidity was still high, the free $\mathrm{HCl}$ being 0.18 per cent. and the total acidity 63 (these being figures after the perforation had been successfully treated). The perforation occurred in a similar site in both instances-namely, in the jejunum just distal to the line of the anastomosis and on the anterior surface, therefore being truly jejunal.

In the subsequent follow-up and investigation of the first case the free $\mathrm{HCl}$ was 0.09 per cent. rising to 0.20 per cent., and the total acidity 60 rising to 84 , and gastroscopy also showed hyperplastic gastritis with secreting mucosa in the remaining portion of the stomach. The actual perforation is of course only a complication of the persisting ulceration, but since partial gastrectomy was accepted as a treatment for duodenal ulcer cases with persistence of symptoms after it have been accumulating. The two cases reported suggest that if a partial gastrectomy must be done for a duodenal ulcer it should be a high one, and that a more radical or subtotal gastrectomy should be performed primarily in these cases of chronic duodenal ulcer with high acidity and hyperplastic gastritis rather than the less complete partial gastrectomy, the aetiological factor in ulcer production-namely, excess acid-being associated usually with a hyperplastic gastritis. Other aetiological factors, such as the ulcer diathesis with a fairly high family incidence, infection, poor surgical technique, and lack of medical care, undoubtedly may also play a part, but these as a rule are either not so prominent or can be more easily excluded. Little could be found in the literature with regard to perforation of anastomotic or jejunal ulcers into the peritoneal cavity following partial gastrectomy.

The incidence of gastro-jejunal ulcer following gastrojejunostomy varies from 4 per cent. (Walton) to 24 per cent. (Strauss). After resection these figures fall considerably: 0.4 per cent. (Schwarz) and 10 per cent. (Gatewood). Gastro-jejunal ulcer usually occurs in the efferent loop near the anastomosis or actually at the anastomosisjejunal 25 per cent. and marginal 75 per cent. (Starlinger). Penetration into neighbouring organs is more common than perforation, the incidence according to Starlinger being 5 to 6 per cent. in 2,500 cases of gastro-jejunostomy and 3 to 5 per cent. in 300 cases of resection.

Over the same ten-year period at the London Hospital the records showed five cases of gastro-colic fistula following partial gastrectomy, and two cases of peri-anastomotic abscess the result of slow perforation or leakage. Following gastro-jejunostomy twenty-four cases of gastrocolic fistula are recorded. Marshall and Kiefer state that post-operative jejunal ulcer can also occur after partial gastroresection, but does so with much less frequency than after partial gastro-enterostomy. Symptoms are usually severe, and intractable to medical treatment; there is also a tendency to bleed and to perforate into the abdominal cavity or colon. It is the general experience of those authors that post-operative ulcer is associated with persistently high gastric acidity.

Burke in his discussion of the operative mortality and morbidity of partial gastrectomy for gastric and duodenal ulcer in 606 consecutive cases gives a mortality rate of 5.44 per cent., but no case of perforation of an anastomotic or jejunal ulcer following this operation.

Lake mentions one case of perforated jejunal ulcer in a series of 198 followed-up cases of gastric and duodenal ulcer treated by partial gastrectomy. Such a complication, therefore, is an uncommon one, and its incidence in cases of chronic duodenal ulcer is likely to be kept low by the performance of partial gastrectomy at a level high enough to ensure definite diminution in the free $\mathrm{HCl}$ production and the associated area of hyperplastic gastritis in the stomach wall. Unfortunately this is associated with a higher primary operative mortality, and in some cases where there is a real ulcer diathesis ulcers tend to recur, whatever the treatment.

I am most grateful to Mr. E. C. Lindsay and Mr. A. C. Perry for permission to publish their cases and for their advice and suggestions; also to Sir James Walton for his help with the bibliography, and to Mr. Hermon Taylor for his gastroscopic report and criticism.

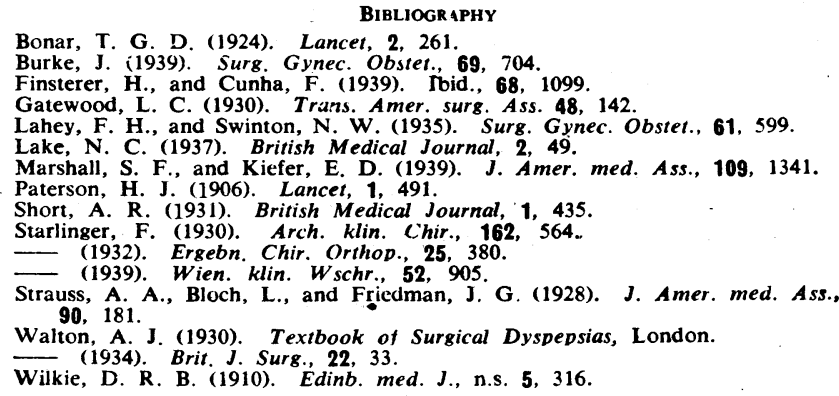

\section{USE OF THE THIERSCH SKIN GRAFT}

\author{
BY
}

B. K. RANK, M.S.Melb., F.R.C.S.

(From the Plastic Surgery Unit of the London County Council -St. James Hospital)

The rapid healing of any raw surface is important in minimizing a patient's discomfort and invalidity, but above all in obtaining the earliest and maximum return of function. Most raw surface areas are produced by accident or surgery. Skin grafting is the most prompt available method of obtaining healing of such an area, and it is the one which produces the least subsequent distortion. The general surgeon does not use skin grafting to full advantage. This can only be explained by a widespread unfamiliarity not only with its technique but with the results to be expected from its application. The Thiersch graft is the one most universally applicable, but certain conditions must be laid down for its use. If these, together with details of technique, are respected the results are good; if not they are correspondingly bad.

This paper is based on a review of all the Thiersch grafts done by the plastic surgery unit of the London County Council.* Special consideration has been given to cases in which the grafts partially or completely failed. Attention to various points will help to make the procedure of skin grafting more certain in the hands of a general surgeon. It may then be used as a prime method of treatment rather than as a last resort. It is significant that many of the conditions treated could have been dealt with more effectively by grafting at a much earlier stage in their clinical history. Moreover, primary skin grafting would have altogether prevented many of the gross deformities and much of the malfunction which have been seen.

* In all, 629 grafts ; these include 535 surface grafts and ninetyfour inlay grafts. Thirty others were excluded because of inadequate records. 


\section{Nomenclature and Technique}

A true Thiersch graft-as used and described by Thiersch and Ollier-is an extremely fine "tissue-paper" epidermal graft which is now rarely employed for general purposes. What is commonly called a Thiersch graft is a thicker graft variously designated by the synonyms "split skin graft," "intermediate graft," and "dermo-epidermal graft." Gillies recommends the use of the term "razor graft " to distinguish these grafts from a "dissected graft" ("full thickness" or Wolfe graft). In this article the term "Thiersch graft" is used in its loose sense for all grafts cut with a razor.

The technique employed has been uniform in principle. It is essentially that evolved during and after the Great War by the Sidcup School of Plastic Surgery, where the Esser principle of pressure-grafting was applied and elaborated. It is well described by Kilner. (Details are considered later.)

\section{Results Obtained}

The conditions for which Thiersch grafts were used and the results obtained are analysed in Table I. Owing to a

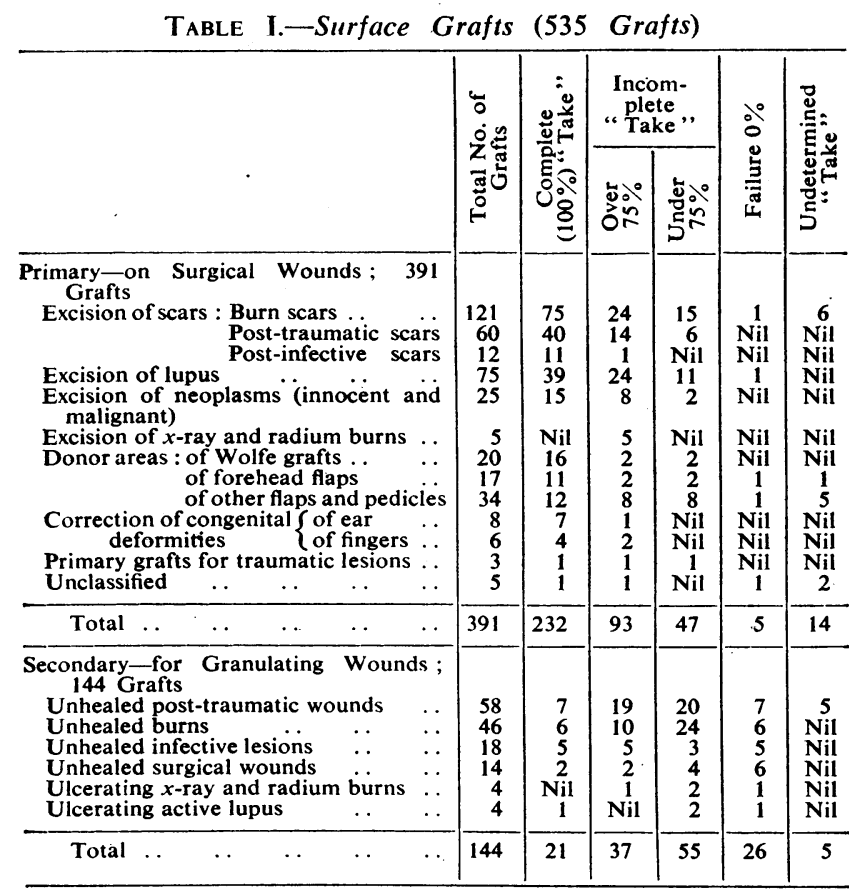

vast difference in prognosis, grafts on newly created surgical wounds (primary grafts) are here considered separately from grafts on long-standing (unhealed) or granulating areas (secondary grafts). The good results of primary grafts contrast with the poor results of secondary grafts.

Primary Grafts.-The results of 391 Thiersch grafts applied to surgically created surface wounds (Table II) show that in 83 per cent. of cases the "take" was either complete or good. These figures do not include inlay grafts, which are even more certain in their " take."

TABLE II

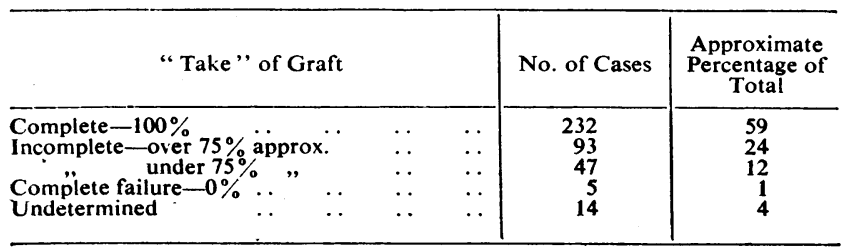

A recently created and sterile raw tissue surface is the ideal base for a Thiersch graft. In this series nearly all the primary grafts were to surgical wounds produced by the eradication of a scar or disease focus. Recently created accidental wounds, after careful preparation and judicious excision of contaminated and devitalized tissue, provide the same ideal basis for a Thiersch graft. The wide-spread impression that skin will only "take" on fatty or fibrous tissue is quite erroneous: skin will do so on all tissue likely to be presented, with the exception of cartilage, bone devoid of periosteum, and tendon denuded of its sheath. The "take" is always exceedingly good on muscle. The good results of primary grafting should dispel any fear of creating a large raw surface area if the latter is thought necessary to the eradication of a disease, and any diffidence in dealing with extensive traumatic skin loss.

Secondary Grafts.-The results of 144 grafts applied to unhealed or granulating areas are given in Table III. In only 41 per cent. of cases was the "take" good or complete. Sepsis is the outstanding cause of these relatively poor results.

T.BBI F. III

\begin{tabular}{|c|c|c|c|}
\hline "Take" of Graft & & No. of Cases & $\begin{array}{l}\text { Approximate } \\
\text { Percentage of } \\
\text { Total }\end{array}$ \\
\hline Complete-100\% $75 \%$ approx. & $\begin{array}{ll}\cdots & \cdots \\
\cdots & \cdots\end{array}$ & $\begin{array}{l}21 \\
37\end{array}$ & $\begin{array}{l}14 \\
27\end{array}$ \\
\hline 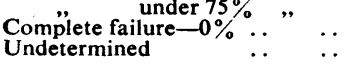 & $\begin{array}{l}\cdots \\
\cdots \\
\cdots\end{array}$ & $\begin{array}{r}55 \\
26 \\
5\end{array}$ & $\begin{array}{r}38 \\
18 \\
3\end{array}$ \\
\hline
\end{tabular}

\section{Causes of Graft Failure and their Prevention}

SEPSIS

In 72 per cent. of all cases. in which the "take" was not complete, failure was attributable to surface sepsis. These infections are usually of low grade and cause little disturbance of the patient's general condition. Mixed infections by the pyogenic organisms-staphylococcus, streptococcus, $B$. pyocyaneus, and $B$. proteus - are usually present. The failure is due to the mechanical separation of the epithelium from its new bed by pus accumulation. Pure streptococcal infections associated with local erysipeloid reactions about a grafted area but not with pus formation usually do not affect the "take" of a graft. Graft failure is related to the amount of surface pus formation and not to the virulence of the organism.

A Thiersch graft placed on a weeping or purulent granulation will fail. Unhealthy septic granulations are characteristically hypertrophic, pale, and mushy. Granulation tissue can rarely be rendered sterile, but before grafting is carried out the infection can be reduced to a minimum by combining two procedures: (a) pre-operative treatment of the granulating area, and $(b)$ removal of the granulation surface before applying the graft.

(a) Treatment of a Granulating Area.-Prolonged and frequent hypertonic saline baths are the most valuable aid in reducing surface infection. These should be used whenever the site of the lesion and the patient's general condition permit. Of local dressings the hypochlorites are the most generally useful. Regulated exposure of the granulating area to ultra-violet light, protected by a layer of paraffin gauze, helps to reduce surface discharge. Pyocyaneus infection is rapidly cleaned by dressing with 2 per cent. acetic acid or household vinegar. The importance of the patient's general condition cannot be overemphasized. Debilitated subjects attending as hospital out-patients with unhealthy granulating wounds rapidly 
improve with in-patient treatment. This especially applies to lesions on the legs.

Under adequate treatment most granulating surfaces show a rapid diminution in the amount of exudate. The granulations become firm and of a bright pink colour, and even though surface cultures still grow organisms ( $B$. proteus is the last to disappear) the areas can be grafted with a much greater chance of success. Antiseptics generally are inimical to skin growth, so it is important that the area be washed and dressed only with saline for two days preceding grafting.

If an unhealthy state of the granulations persists it is usually because of an underlying focus of infection-for example, sloughing tendon or bone sequestration. If there is no such obvious cause for the persistence of unhealthy granulations, and especially if previous grafts have failed, one must always consider the use of "pinch" grafts. Pinch grafts offer the only hope of success in such conditions, as exudate is able to escape without mechanical separation of the "pinches." In this way epithelization might be sooner obtained than it would be by waiting until conditions are adequate for the use of a Thiersch graft.

(b) Removal of the Granulating Surface.-In a further attempt to increase the relative sterility of the recipient area it has been the practice, where possible, to excise the area of an unhealed wound or remove granulation tissue by curettage. The graft is then placed on the newly created fibrotic but vascular base. As shown in Table IV, the results of grafts on areas so treated have been slightly better than in areas where the granulations were untouched. Moreover, less fibrosis is developed beneath these grafts, which consequently have a much better texture.

TABLE IV

\begin{tabular}{|c|c|c|c|}
\hline "Take" of Graft & & $\begin{array}{l}\text { Granulations } \\
\text { l.eft in situ }\end{array}$ & $\begin{array}{c}\text { Granulations } \\
\text { Removed }\end{array}$ \\
\hline $\begin{array}{l}\text { Complete-100\% } \\
\text { Incomplete-over } 75 \% \text { approx. } \\
\text { complete failure- } 75 \% \%, \quad \ldots\end{array}$ & $\begin{array}{ll}\cdots & \cdots \\
\cdots & \cdots \\
\cdots & \cdots\end{array}$ & $\begin{array}{r}6(12 \%) \\
11(23 \%) \\
22(46 \%) \\
9(19 \%)\end{array}$ & $\begin{array}{l}15(16 \%) \\
26(28 \%) \\
34(37 \%) \\
17(19 \%)\end{array}$ \\
\hline Total number . . & . & 48 & 92 \\
\hline
\end{tabular}

\section{HAEMATOMAT}

After pus, blood is the most common cause of mechanical separation of the epithelium from its bed, resulting in complete failure of the graft or, more usually, a "patchy take." In 2 per cent. of the grafts of this series partial or complete failure was due to a haematoma beneath the graft. In most cases the haematoma was associated with infection. Most haematomas occurred under grafts to surgical wounds of the face, where local vascularity always makes absolute haemostasis more difficult. Absolute haemostasis on a graft-bed is essential, and it must be ensured by clamping and, if necessary, tying off major points, or by the pressure of hot and cold packs. Before fixing the pressure-dressing on a large graft where haemostasis has been difficult it is a wise precaution to syringe beneath the graft with normal saline from a dental syringe. Once haemostasis is established an effective pressure-dressing will ensure its persistence.

\section{INADEQUATE FIXATION OF THE GRAFT}

In only seven cases of this series was partial (five cases) or complete failure (two cases) directly attributable to inadequate fixation of the graft. In inexperienced hands this is a common cause of graft failure. The correct fixation of a graft and the application of the necessary pressure-dressing require patience. In general the graft is spread on tulle gras* and, with it, is sutured to the circumference of the wound, some of the anchoring stitches being left of sufficient length to tie over and so maintain the pressure-dressing. In some cases the skin is applied free to the wound. The graft should be so arranged as to cover the whole defect. Pressure may be applied by a stent mould: this is the method of choice for inlay grafts (the graft is introduced spread on the mould), but it has generally been abandoned for surface grafts owing to the ease with which it may shift. On surface grafts, wool soaked in equal parts of paraffin and acriflavine 1 in 1,000 , wrung out and built up evenly over the area in flat plaques, is the usual dressing. The contour is made even, gauze pressure is applied, and the dressing is then tied to the area by the long ends of the anchoring sutures. Marine sponge fixed in the same way is used in some regions-it may be combined with paraffin and acriflavine wool. It is important to see that the pressure-dressing bulges over the edge of the graft, as most of the partial failures without obvious cause show defects about the circumference of the graft where the fixation and pressure have been inadequate.

The pressure-dressing is fixed by a firm crêpe bandage over wool, by elastoplast, or by "lastonet" and mastisol, according to the region. Mastisol is very useful in helping the fixation of all dressings. Limb splintage may be required.

Inlay grafts (cavity grafts), where the fixation and pressure are firmly maintained by a gutta-percha or stent mould, have universally given good results. Grafts on the forehead "take" exceedingly well owing to the ease of maintaining firm pressure-fixation against the skull. Conversely, about joints, on the chest and axilla, and on the neck, absolute fixation is always more difficult, and it is in these regions that most failures from inadequate fixation occur.

The primary pressure-dressing is left undisturbed for at least seven days. Though a graft "takes" sooner than this, and the dressing may be removed in about five days with success, the graft is still unstable on its new bed and may become separated. If, however, there is evidence of sepsis and discharge on the dressing it is wise to remove the pressure-dressing carefully after five days, as there may be areas of vital skin under which pus has not yet caused mechanical separation. A fresh pressure-dressing can then be reapplied to these areas, and by this means a graft which would otherwise be a failure is often preserved as a "patchy take."

\section{Graft and Donor Area}

Strict aseptic preparation of the donor area is important, and even the most trivial skin infections contraindicate its use as a graft. The thinner the graft is cut the more certain is its "take." If the graft is cut too thick, to include "full thickness" areas, not only is the prospect of "take" reduced but delayed healing by secondary intention is caused in the donor site. This increases the risk of bad scarring, with keloid formation. The donor area of a well-cut graft (usually the posterolateral aspect of the thigh) should require only its primary

* Tulle gras is a large-mesh gauze $(2 \mathrm{~mm}$.) soaked in a mixture of petroleum jelly and balsam of Peru (1 per cent.). As made by the Anglo-French Drug Company, "tulle gras lumière " the Anglo-French Drug Company, "tulle gras lumière also contains 1 per cent. halibut-liver oil. Introduced by Gillies to skingrafting, it has eliminated the practical difficulties of spreading
and placing the graft, as the latter is very easily spread and handled and placing the graft, as the latter is very easily spread and handled
on tulle gras. An effective substitute can be made in hospital from a coarse-mesh cotton gauze soaked in petroleum jelly and 1 per cent. balsam of Peru and sterilized in an autoclave. 
dressing, which can be discarded in a week. It should heal without scar. Very often the donor sites are subsequently reversed. Though theoretically a Thiersch graft should contain no hair follicles, hair is often noted on grafted areas, especially if large. Hair growth may be very undesirable in certain regions, especially with inlay grafts. The inner aspect of the arm is the most useful non-hairy donor site.

\section{Recipient Region}

A study of the results in relation to the region grafted reveals that these are uniformly better in regions to which absolute fixation and firm pressure are easily appliedfor example, the forehead, the upper face, and nonarticular regions of the limbs. Otherwise there is no indication that grafts "take" better on some regions than on others. It seems that the local blood supply does not markedly affect the primary "take" of a Thiersch graft. The subsequent behaviour and stability of a graft are, however, greatly influenced by the local blood supply. A graft may "take" well on the area of a large chronic ulcer of the leg, only to break down again when walking is resumed. Thiersch grafts on the sites of old $x$-ray burns with their poor local vascularity often prove unstable and develop areas of breakdown and ulceration.

\section{Conclusions}

The results of primary Thiersch grafts applied to surgical wounds are uniformly good, as the potential causes of graft failure-sepsis, haematoma, and inadequate pressure-fixation-are all minimized by careful technique.

The results of Thiersch grafts applied secondarily to unhealed areas are by far inferior to the above because of the increased incidence of sepsis. This can be reduced by a period of careful preparation of the granulating area and, when practicable, removal of the potential infection by excision of the area or by scraping off the granulations.

By a wider and careful application of skin grafts to recent wounds many of the problems of secondary grafts to granulating areas could be eliminated, much prolonged invalidity would be reduced, and better functional results often obtained in a shorter period. Much of the ugly scarring, with bad contractures and deformities, which has been treated in this series would never have arisen.

I wish to thank the medical officer of health to the London County Council, and the medical superintendent of St. James Hospital, for permission to publish this article. I am indebted to Sir Harold Gillies, Mr. A. H. McIndoe, and Mr. Rainsford Mowlem for their kindly help and criticism.

\section{ReFERENCE}

Kilner, T. P. (1934). Post-grad. med. J., 10, 176.

The Royal London Ophthalmic Hospital, better known as "Moorfields," has carried on in spite of the dislocation due to the war and the necessity for placing over eighty beds at the disposal of the Ministry of Health for air-raid and Service casualties. The annual report states that a complete ophthalmic service was afforded during 1939. At Moorfields itself eighty-eight beds were retained, and fifty-three were made available at St. Mary-at-the-Cross Hospital, Edgware-a total of 141 for civilian eye patients as compared with a normal complement of 200. The number of in-patients in 1939 was 3,636 , and of out-patients 53,108 . The report includes an analysis of the diseases and injuries of the in-patients, and of the operations performed in the in-patient and out-patient theatres. The hospital accounts show an excess of total income over total expenditure of $£ 5,822$.

\section{BARBITURATE (SONERYL) POISONING} AN ACCOUNT OF A CASE SHOWING THE
VALUE OF PICROTOXIN IN TREATMENT BY

\author{
GERALD M. SLOT, M.D., M.R.C.P., D.P.H. \\ Senior Physician, Royal Waterloo Hospital, and Royal \\ Hospital, Richmond; Consultant, L.C.C. and Emergency
}

Medical Service

I have seen a number of cases of barbiturate poisoning, which is becoming increasingly common. This is perhaps because barbiturates are widely used and can easily be obtained in spite of the prescribing restrictions. Large doses to cover a period are often prescribed, especially in out-patient hospital work, and by applying to one or more medical men a patient can easily obtain a store without their knowledge. It is impossible in most cases to know accurately the dose that has been taken. The outstanding features are coma, low blood pressure, respiratory failure, and in many cases a dusky colour.

\section{Case Record}

On January 17, 1940, a young woman of 26 went to bed at her hotel. The following morning the maid attempted to enter the room, but received no reply; after some delay an entry was forced and the woman was seen to be in deep coma. The hotel doctor was summoned and he found two empty bottles at the side of the bed. The chemist's name was on the bottles, and investigation proved that each bottle had contained twenty-five tablets of soneryl, a barbiturate which acts less rapidly than the pentothal group but far more rapidly than phenobarbitone. The prescription for the drug was issued by another doctor in September, 1939, but was not brought for dispensing until January this year. It is difficult to know exactly what dose was taken-it was at least 37 grains and at most 74 grains. From the date of dispensing, assuming normal use previously, perhaps 65 grains might be a fair estimate.

The patient was removed to a nursing home. On admission her pupils were small and non-reacting and all her reflexes were absent. Her colour was dusky, the pulse small and thready, and the blood pressure $85 / 50 \mathrm{~mm}$. Hg. Breathing was stertorous, and frothy blood-stained mucus came from the mouth at intervals. The blood in this case may have come from the tongue, which was bruised by tongue forceps at the first attempt to secure an airway.

\section{TREATMENT}

The immediate treatment was: (1) injection of coramine ; (2) washing out the stomach and filling with three-quarters of a pint of strong black coffee and $1 \mathrm{oz}$. of castor oil ; (3) two-hourly injections of cardiazol; (4) catheterizing the bladder (urine was normal); (5) initiation of a glucose-saline drip, 5 per cent., with a vacoliter apparatus: the drip was regulated 18-20 drops a minute to avoid "wet" lungs. The treatment was kept up for six to eight hours, but the patient's condition deteriorated. Her pulse grew feebler and the breathing more laboured. The outlook was gloomy Later that evening a lumbar puncture was done and $10 \mathrm{c.cm}$ of clear cerebrospinal fluid not under pressure was removed. An airway was inserted, and this helped the breathing considerably. Owing to lack of supply, cardiazol was substituted by anacardone, and $1 \mathrm{c.cm}$. of this was given two-hourly.

The next morning the coma was as deep as previously and a further lumbar puncture was done. On this occasion only $1 \mathrm{c} . \mathrm{cm}$. of clear lumbar fluid came away. It may have been that this was due to low blood pressure and little was being secreted. Later that day she was again catheterized and $10 \mathrm{oz}$. of urine was withdrawn. This showed that the kidneys were still functioning to some extent. Her temperature rose to $102^{\circ}$, and the pulse, still thready, fluctuated between 110 and 120 . Her blood pressure rose to $100 \mathrm{~mm}$. after the injection of anacardone, but fell to $85 \mathrm{~mm}$. within an hour. 